\title{
Efecto de la Intervención Docente en la Percepción de Competencia y Motivación de Futuros Maestros de Primaria en Educación Física Utilizando la Evaluación Formativa y Compartida
}

\author{
The Effect of a Teaching Intervention on Pre-service Primary School Teachers' \\ Perceived Competence and Motivation in Physical Education \\ Utilizing Formative and Shared Assessment
}

Isaac Estevan ${ }^{a}$, Javier Molina-García ${ }^{b}$, Xavier García-Massóc, Daniel Martos ${ }^{d}$

\author{
${ }^{a}$ Universidad de Valencia, Telf.: (34) 961625109 \\ Correo electrónico: isaac.estevan@uv.es \\ ${ }^{\text {b } U n i v e r s i d a d ~ d e ~ V a l e n c i a, ~ T e l f .: ~(34) ~} 961625170$ \\ Correo electrónico: javier.molina@uv.es \\ ${ }^{c}$ Universidad de Valencia, Telf.: (34) 963864694 \\ Correo electrónico: xavier.garcia@uv.es \\ ${ }^{\mathrm{d}}$ Universidad de Valencia, Telf.: (34) 963983383 \\ Correo electrónico: daniel.martos@uv.es
}

\begin{abstract}
RESUMEN
El objetivo del presente estudio fue analizar el efecto percibido de una intervención docente en el área de Educación Física basada en evaluación formativa y compartida. Se realizó una intervención a 340 participantes voluntarios (239 mujeres), divididos en grupos control $(n=206)$ e intervención $(n=134)$. Antes y después de la intervención se midió su percepción de competencia en evaluación y motivación hacia la Educación Física. Se halló un efecto principal de la intervención sobre la motivación del alumnado $(p<0,001)$, pero no sobre la percepción de competencia $(p>0,05)$. Tras el periodo docente, el grupo intervención aumentó su motivación mientras que el grupo control la disminuyó. Como conclusión, una intervención docente centrada en metodología activa-participativa e inclusión de evaluación formativa y compartida favorece que los futuros egresados mejoren su motivación hacia los contenidos de la asignatura de Educación Física, estableciéndose una relación causal.
\end{abstract}

Palabras claves: innovación docente, metodología, formación egresados, universidad.

\begin{abstract}
The purpose of this study was to examine the perceived effect of a physical education teaching intervention by applying formative and shared assessment. The experiment was applied to 340 volunteers ( 239 women), divided into control $(n=206)$ and intervention $(n=134)$ groups. A test and a retest (after the trial) were conducted to assess perceived competence in assessment and motivation in physical education. The intervention was found to have a significant effect on motivation $(p<0.001)$, but not on perceived competence $(p>0.05)$. After the teaching period, an increase in the motivation of the intervention group and a decrease in the motivation of the control group were noted. We conclude that a teaching intervention focused not only on active and participative methodology but also on formative and shared assessment facilitated an increase in motivation toward course contents in physical education for future teachers, establishing a causal relationship.
\end{abstract}

Keywords: teaching innovation, methodology, teacher training, university. 
Estudios Pedagógicos XLIV, N $^{\circ}$ 2: 205-221, 2018

EFECTO DE LA INTERVENCIÓN DOCENTE EN LA PERCEPCIÓN DE COMPETENCIA Y MOTIVACIÓN DE FUTUROS MAESTROS DE PRIMARIA EN EDUCACIÓN FÍSICA UTILIZANDO LA EVALUACIÓN FORMATIVA Y COMPARTIDA

\section{INTRODUCCIÓN}

Desde el año 1999 en que la Declaración de Bolonia inicia el proceso de convergencia europea en materia de educación, se han venido desarrollando paulatinamente los cimientos del llamado Espacio Europeo de Educación Superior (EEES). Dicho proceso ha mantenido diversos objetivos, como el de unificar un sistema europeo que facilitase el tránsito y el intercambio, la focalización de la atención docente en el proceso y el alumnado, es decir, en el aprendizaje, o el aumento de la calidad de la educación y, por ende, plantar cara al súper competitivo sistema norteamericano (Pérez-Pueyo, Julián \& López-Pastor, 2009). Esto ha llevado a determinar cómo, a pesar de las críticas que el EEES ha suscitado, nos encontramos ante una oportunidad única de transformación de los sistemas educativos. En este sentido, parece obvio que el avance de las sociedades modernas, al calor, por ejemplo, de los descubrimientos tecnológicos, obliga a reformular los postulados educativos para atender las dinámicas necesidades sociales. Así, los sistemas educativos de muchos países se han reformado para dar cabida a las nuevas formas de entender los procesos de enseñanza y aprendizaje, entre otros motivos, por el advenimiento de las llamadas sociedades de la información y la comunicación (Sebastiani, Blázquez \& Barrachina, 2010).

Uno de los pilares del cambio suscitado en las estructuras educativas europeas ha sido el establecimiento del sistema de competencias. En el estado español, la actual Ley Orgánica 8/2013, de 9 de diciembre, para la mejora de la calidad educativa (LOMCE), lo explica claramente: "Las habilidades cognitivas, siendo imprescindibles, no son suficientes; es necesario adquirir desde edades tempranas competencias transversales" (p. 97860). Las competencias se pueden entender como "una combinación de conocimientos, capacidades y actitudes adecuadas al contexto" (Diario Oficial de la UE, 2006), y que buscan mejorar las respuestas de cada persona a las demandas de cada situación por lo que, en palabras de Sebastiani et al. (2010) se pretende acercar lo que se aprende en la escuela a las exigencias de la vida moderna. Esta pretensión, que ha modificado no solo los currículos escolares de todas las áreas ya desde la Ley Orgánica de Educación (2006), sino también el diseño de los títulos de grado universitarios, ha sido objeto de duras críticas. En palabras de Vicente (2011), el modelo formativo basado en competencias tiene su origen en el mundo de la empresa y se aferra a la lógica de la economía y la rentabilidad, aunque adaptado al escenario pedagógico.

De todas formas, más allá de las críticas, la presencia e influencia de las competencias en la educación, en general, y en la educación física (EF), en particular, es ya una realidad (Figueras et al., 2016). A pesar de no contar con una competencia motriz, específica de nuestra área, la EF puede contribuir, en mayor o menor medida, a la consecución de todas las competencias establecidas en los currículos escolares (Méndez, López-Téllez \& Sierra, 2009). Sin embargo, la implantación de un modelo formativo basado en competencias no está siendo un proceso agradable, a tenor de las barreras y dificultades encontradas. En este sentido, el sistema de competencias parece no estar bien detallado y redactado, es un añadido a las normas anteriores y/o no acaba de convencer al profesorado (Monarca \& Rappoport, 2013), entre otros factores. En EF el panorama no es más halagüeño, a tenor de las múltiples barreras que el profesorado percibe en su desarrollo (Zapatero-Ayuso, González-Rivera y Campos-Izquierdo, 2017), la falta de unas directrices claras que guíen su implantación curricular (Figueras et al., 2016) o la superficialidad con que se tratan las competencias en las programaciones (Hortigüela, Abella \& Pérez-Pueyo, 2015). 
Si obviamos las críticas al sistema de competencias y asumimos que su implantación es una necesidad educativa o, al menos, una obligación legal, habremos de profundizar en su estudio y evaluación. En esta tesitura, la opinión del alumnado se revela importante; este es uno de los objetivos de este estudio. Concretamente, se pone atención en alumnado del Grado en Maestro o Maestra de Educación Primaria, es decir, en la formación inicial de los/las docentes. Como ya hemos mencionado, los grados universitarios han asumido el modelo competencial en relación con el establecimiento del EEES (Pérez-Pueyo et al., 2009), y esto afecta a todos los elementos que componen el proceso educativo, también a la evaluación.

La evaluación, en esencia, se puede entender como un proceso "basado en recoger información, analizarla y emitir un juicio sobre ella” (Hamodí, 2016, p. 25), aunque cabe hacer múltiples matices sobre el cuándo, el cómo, el quién o, incluso, el porqué. En este sentido, la evaluación es un proceso complejo y sumamente importante, más allá de un simple añadido o una formalidad, por cuanto su planteamiento afecta de forma fundamental a la mejora de la enseñanza y el aprendizaje (Brew, Riley \& Walta, 2009). Tradicionalmente, en EF se han utilizado y sugerido modelos de evaluación únicamente centrados en la valoración de la condición física (López-Pastor et al., 2013a). De manera alternativa, Siedentop y Tannehill (2000) sugieren la utilización de modelos de evaluación que impliquen al alumnado activamente en la resolución de problemas reales utilizando información, conocimiento y habilidades relevantes. Recientemente se ha puesto de manifiesto que la metodología docente y el sistema de evaluación utilizados influyen no solo en el aprendizaje del alumnado, sino también en su motivación hacia los contenidos tratados y en la percepción de competencia (Hortigüela et al., 2016; Hortigüela, PérezPueyo \& López-Pastor, 2015).

Dentro de los distintos tipos de evaluación, las evaluaciones alternativas responden a formas diferentes de concebir la educación. Así, frente a un modelo de evaluación tradicional, que atiende a la enseñanza y se ocupa básicamente de certificar el éxito o el fracaso del alumnado (López-Pastor, 2009a), estas formas alternativas de evaluación se centran principalmente en el aprendizaje y el alumnado. La aplicación de modelos alternativos de evaluación es un gran reto para el sistema universitario ya que se debe pasar de la calificación tradicional por medio de un único examen a la implicación del alumnado en la comprensión de la evaluación dentro del proceso de aprendizaje (Dochy, Segers \& Dierik, 2002). En este punto, introducimos dos conceptos clave: la evaluación formativa y la compartida. Sucintamente, la primera es aquella que pretende la mejora del aprendizaje mediante el feedback continuo al alumnado, de forma que este sea consciente de lo que hace bien y de lo que debe modificar (Brew et al., 2009), mientras que la segunda hace alusión a la participación de las y los alumnos en su propia evaluación, haciendo uso del diálogo y el acuerdo para la mejora constante (López-Pastor et al., 2011). La participación activa del alumnado en los procesos de evaluación favorece su formación (Tan, 2008). Para buscar la implicación del alumnado en los procesos de evaluación, podemos encontrar algunas estrategias:

- La autoevaluación es aquella que realiza la persona sobre sí misma, en este caso, el alumno o alumna sobre su proceso de aprendizaje, de forma que, siguiendo a Muros (2013), se haga consciente de su responsabilidad y aprenda a darle valor a las tareas desarrolladas. 
- La evaluación por pares o entre iguales es en la que se da una evaluación recíproca entre alumnos o alumnas, pudiéndose ser individual o colectiva.

- Coevaluación se refiere al proceso de evaluación que, de forma dialogada, desarrolla el profesorado con su alumnado.

En los últimos años la literatura sobre evaluación en educación superior ha producido una notable cantidad de estudios, a pesar de no ser la práctica más habitual en las universidades españolas (López-Pastor et al., 2011). Sin embargo, los datos surgidos de estas investigaciones apuntan cómo la evaluación formativa y compartida (EFC) no solo es coherente con los postulados del EEES y el sistema de competencias (Brown \& Glasner, 2003; López-Pastor, 2009a), sino que arroja resultados positivos y de gran valor en la mejora de los procesos de enseñanza y aprendizaje (Trevitt, Brenan \& Stocks, 2012). Así, se trata de una evaluación orientada al aprendizaje (Boud \& Falchikov, 2007; Carless, Joughin \& Mark, 2006), en la que el docente debe ayudar y cooperar con el alumnado para, de esta forma, favorecer el aprendizaje crítico y reflexivo orientado a una mayor autonomía en relación con las responsabilidades propuestas (Brockbank \& McGill, 2002; Dekker-Groen, Van der Schaaf \& Stokking, 2013). Sucintamente, la EFC se refiere a todo proceso de evaluación cuyo fin es mejorar el proceso de enseñanza-aprendizaje por medio del diálogo y la toma de decisiones mutuas y/o colectivas, evitando la imposición y la decisión individual. Además, la EFC aúna la búsqueda de la coherencia y planteamientos epistemológicos y pedagógicos basados en la teoría y práctica educativa (López-Pastor et al., 2006). Asimismo, la EFC estaría relacionada con una racionalidad práctica que está más centrada con los procesos de desarrollo personal y grupal, en contraste a la racionalidad técnica de carácter más instrumental y centrada en el currículum por objetivos (Pascual, 1996). En este sentido, la EFC estaría en consonancia con metodologías de investigación predominantemente cualitativas y de carácter vivencial que se basan, sobre todo, en posicionamientos epistemológicos de la investigación más interpretativos o socio-críticos (Sparkes, 1992). No obstante, se debe tener en cuenta que:

Los modelos y discursos existentes de Evaluación en Educación Física no constituyen entes aislados ni separados, como a veces puede parecer, sino que están fuertemente relacionados con modelos, discursos, paradigmas y racionalidades más amplias y complejas, que son las que les dan sentido y coherencia (o se la quitan) en la práctica educativa cotidiana. (López-Pastor et al., 2006, p. 35).

Es por ello que, hoy en día, son habituales los estudios cuyos diseños de investigación poseen características epistemológicas de tipo positivista con el fin de conocer qué cambios se han producido a partir de la puesta en práctica de un modelo de evaluación basado en una racionalidad práctica, por ejemplo de EFC, como es el caso del presente estudio.

La EFC supone un gran reto docente que nos acerca a los planteamientos del EEES, donde se requiere de sistemas de evaluación coherentes con la utilización de metodologías activas de enseñanza y el desarrollo de competencias profesionales. Así, parece ser que la EFC es una de las metodologías de evaluación más adecuada y coherente en Educación Superior por sus ventajas en los procesos de aprendizaje del alumnado (López-Pastor, 2011). En un estudio sobre evaluación entre iguales, Van der Berg, Admiraal y Pilot (2006) encontraron que el alumnado valoraba positivamente leer y evaluar los trabajos de otros y encontraban útil el feedback recibido. En esta línea, Hortigüela, Pérez-Pueyo y López- 
Pastor (2015) indicaron cómo el alumnado universitario implicado en procesos de EFC tenía una sensación de justicia hacia la calificación obtenida y percibían cómo la evaluación se refería a aspectos variados, más allá de la memorización. En añadidura, López-Pastor (2008) explicitaba que la EFC aumenta la implicación del alumnado en el aprendizaje y, con ello, el rendimiento académico. Estos resultados se han dado independientemente de los estudios de grado implicados; ahora bien, en lo que respecta a alumnos y alumnas de formación del profesorado, como es este caso, parece más relevante, si cabe, promover la participación en procesos de EFC para que, de esta manera, sientan la confianza suficiente para usarlo en el devenir de su labor docente (Brew et al., 2009; Lorente \& Kirk, 2012; Martos-García, Usabiaga \& Valencia-Peris, 2017).

La utilización de sistemas de evaluación orientados al aprendizaje, como la EFC, han resultado eficaces a nivel internacional no solamente en la adquisición de competencias específicas de los contenidos impartidos (López-Pastor et al., 2013a), sino también en el aumento del interés y la motivación por los contenidos trabajados (Hortigüela, Pérez-Pueyo \& Abella, 2015; Mintah, 2003), en el presente caso, los relativos a la EF. La motivación hacia los contenidos de EF ha sido analizada desde diversas perspectivas para conocer la satisfacción de las necesidades psicológicas básicas (i.e., autonomía, competencia y relación) por su relación con los objetivos del área en el ámbito educativo (Moreno et al., 2008). En este sentido, parece ser que valorando la percepción de competencia y la motivación del alumnado objetivamente podemos conocer el grado de cumplimiento de las pretensiones y los objetivos iniciales planteados (Varsavsky, Matthews \& Hodgson, 2014). Así, una de las aportaciones de estudiar la motivación en EF es el índice de autodeterminación, el cual permite ofrecer una estimación de la importancia de orientar al alumnado hacia la mejora personal en tareas concretas, el esfuerzo y la perseverancia, en contraposición a la mera comparación con los demás (Moreno et al., 2008; Moreno, González \& Chillón, 2009). Así, analizar este índice en los futuros docentes parece ser un asunto relevante para conocer cómo afrontarían la intervención para desarrollar sensaciones de competencia, autonomía e interacción grupal en el alumnado. Y es que los docentes de EF que muestran una mayor motivación parecen formarse más en su labor profesional y mostrar amplias competencias docentes (Carson \& Chase, 2009).

De todas formas, no todos los datos tienen una lectura positiva, aunque en general se manifieste la bondad de la EFC en comparación con la evaluación tradicional. Y es que, pese al potencial formativo la EFC, por su novedad y el diferente tipo de trabajo que requiere, puede generar cierta resistencia tanto por parte del alumnado como de las familias o incluso del resto del profesorado (López-Pastor et al., 2006). Así, por poner algunos ejemplos, la EFC hace que el alumnado ( $\mathrm{y}$ en algunos casos el profesorado) perciba una mayor carga de trabajo, sienta que haya poca diferenciación en la calificación entre individuos o manifieste explícitamente su rechazo a participar en estos procesos (Atienza et al., 2016; LópezPastor et al., 2013; Lorente \& Kirk, 2012; Mintah, 2003). Por todo ello, pese a que la EFC favorece el desarrollo de competencias docentes y el aprendizaje del alumnado, en la actualidad todavía son predominantes las universidades españolas que parecen alejarse del uso de estos procesos de EFC (Palacios-Picos \& López-Pastor, 2013) y, consecuentemente, es escasa su aplicación en la función docente en Educación Primaria (Chen, 2005).

Con todo, el objetivo del presente estudio fue analizar el efecto percibido por alumnado del Grado en Maestro y Maestra de Educación Primaria de una intervención docente en el área de Educación Física basada en EFC. 


\section{METODOLOGÍA}

\subsection{PARTICIPANTES}

En el estudio participaron 340 alumnos/as (239 mujeres), de segundo ( $n=51,8 \%$ ) y tercer $(n=47,2 \%)$ curso de grado universitario, con una edad media de 21,59 años $(D . T .=2,58)$ que cursaban una asignatura relacionada con la didáctica de la EF y cuya carga lectiva era de 60 horas presenciales (i.e., Didáctica de la Educación Física en Educación Primaria y Didáctica de las Habilidades Perceptivo Motrices y Básicas). Todos/as los participantes firmaron voluntariamente un documento de consentimiento informado, tanto al inicio como al final del estudio. El departamento de formación permanente de la Universidad aprobó el diseño y realización del estudio.

\subsection{DISEÑO DEL ESTUDIO}

Se realizó un diseño cuasi-experimental en 11 grupos del Grado de Maestro o Maestra en Educación Primaria de la Facultad de Magisterio de una única universidad española. Las intervenciones con los grupos se realizaron durante un semestre a lo largo de 2016 y 2017. La asignación de los grupos naturales a cada uno de los grupos de estudio (5 de intervención y 6 de control) fue aleatoria según si recibían la intervención docente en EFC $(n=134)$ o si no la recibían $(n=206)$.

El primer día de clase, tras la explicación de los contenidos de la asignatura, así como la metodología docente, el funcionamiento del grupo y la propuesta de evaluación, el alumnado respondió al inventario de valoración. Tras el proceso de enseñanza-aprendizaje (intervención que a continuación se describe), el alumnado respondió al mismo inventario. La única diferencia entre ambos inventarios fue el tiempo verbal utilizado, futuro y pretérito perfecto, respectivamente.

\subsection{PROCEDIMIENTO}

En ambos grupos, control e intervención, la metodología docente se caracterizó por mantener una orientación de implicación participativa y activa del alumnado. En este sentido, el alumnado debía afrontar retos tales como el estudio y desarrollo de contenidos y su exposición, o la corrección de los trabajos y/o propuestas del resto de compañeras y compañeros. La única diferencia entre los grupos control e intervención fue que a estos últimos se les aplicó la EFC.

El proceso de EFC compartió los siguientes criterios: 1) Previo al inicio de la intervención docente, el profesorado y el alumnado discutieron y llegaron a un acuerdo sobre los valores porcentuales de los diferentes apartados de la evaluación de la asignatura; 2) Además, la evaluación mantuvo tres pilares fundamentales: la autoevaluación, la evaluación por pares y la evaluación compartida o colaborativa (Berk, 2005; Dochy et al., 2002; López-Pastor, 2009a, 2011).

Para su desarrollo se utilizaron:

i. Autoevaluación: entrevistas individualizadas y/o cumplimentación de formularios del alumnado. 
ii. Evaluación por pares: entrevistas individualizadas y/o cumplimentación de rúbricas de evaluación (estas últimas, proporcionadas por los compañeros/as responsables del trabajo y supervisadas por el profesorado).

iii. Entrevistas grupales y/o individualizadas.

3) En los trabajos grupales puntuables en la calificación, los grupos discutieron las calificaciones repartiendo el total de la puntuación de acuerdo a la implicación de cada miembro en el proceso (e.g., un grupo de cinco miembros calificado por el profesor con un 8 sobre 10, disponía de 40 puntos a repartir entre todos. El reparto final debía conseguirse por consenso total de los miembros. En todos los casos, el alumnado llegó a un consenso).

Durante la aplicación de la EFC, el profesorado y alumnado mantuvo entrevistas periódicas, individualizadas y/o por grupos, con el fin desarrollar el proceso de evaluación correctamente. En estas reuniones, entre otras, se explicaron aspectos tales como: el desarrollo de rúbricas de evaluación y escalas de valoración vinculadas a las rúbricas, aportación de retroalimentación de las tareas realizadas y discusión/negociación de evaluaciones realizadas (e.g., autoevaluación y evaluación por pares). Por otra parte, en los grupos control, el profesorado estableció los porcentajes de evaluación y la calificación de los diferentes apartados.

\subsection{INTRUMENTOS}

Se utilizaron diferentes cuestionarios para la valoración de la percepción del alumnado, así como preguntas individuales para disponer de información referente al conocimiento del alumnado sobre las características de la intervención docente en EF. Concretamente, las herramientas utilizadas fueron las siguientes:

La percepción de competencia docente en evaluación en EF se valoró con un cuestionario desarrollado ex profeso para el presente estudio. Estaba compuesto por $22 \mathrm{de}$ las competencias que el alumnado egresado del Grado de Maestro o Maestra en Educación Primaria debe adquirir a lo largo de sus estudios. El cuestionario preguntaba en qué grado creen las y los estudiantes que desarrollan las diferentes competencias docentes mostradas en esta asignatura de EF. El cuestionario fue revisado por dos expertos en la materia de EF y la docencia universitaria. Un ejemplo de los ítems que lo forman es: "Tener capacidad de reflexión sobre el proceso de enseñanza/aprendizaje, los diferentes tipos organizativos y las distintas metodologías dentro de las clases de Educación Física”. Las respuestas a cada uno de los ítems mantenían una estructura tipo Likert entre 1 y 5, siendo 1 "Nada" y 5 "Mucho". La consistencia interna de las respuestas de los participantes antes y después del proceso de enseñanza aprendizaje, medida con el Alfa de Cronbach, fue de 0,89 (IC 95\%, $0,87-0,90)$ y de 0,90 (IC 95\%, 0,88-0,91), respectivamente.

La motivación del alumnado en las clases de EF se midió con una adaptación de la escala de las necesidades psicológicas básicas en EF (Moreno-Murcia et al., 2008). El cuestionario está compuesto por 20 ítems cuyas respuestas mantienen una estructura tipo Likert entre 1 y 5, siendo 1 "Muy en desacuerdo" y 5 "Muy de acuerdo". Aunque la escala está formada por diferentes subescalas, en el presente trabajo se calculó el índice de autodeterminación originalmente propuesto por Vallerand y Rousseau (2001), que también utilizan Moreno-Murcia et al. (2008, 2009). La consistencia interna de las respuestas de 
los participantes antes y después del proceso de enseñanza aprendizaje, medida con el Alfa de Cronbach, fue en ambos casos de 0,79 (IC 95\%, 0,76-0,82).

El grado de acuerdo del alumnado en el planteamiento docente de la asignatura como, por ejemplo, la negociación del sistema de evaluación, la metodología, la adquisición de competencias, el grado de satisfacción y la utilidad de los contenidos, se valoraron con cinco cuestiones independientes cuyo enunciado fue: 1) “ ¿Se ha negociado la utilización del sistema de evaluación en la asignatura al comienzo del curso?”, 2) “¿Crees que la metodología de esta asignatura te ayudará a adquirir competencias profesionales?", 3) “¿La evaluación que se ha planteado favorecerá la adquisición de las competencias profesionales?", 4) "Señala el grado de satisfacción global que crees tendrás en relación con el sistema de evaluación de esta asignatura”, 5) "¿Consideras útiles los contenidos de esta asignatura?". La respuesta a estas cuestiones mantuvo una estructura de tipo Likert entre 1 y 5 , siendo 1 "No/Nada" y 5 "Mucho". El alumnado también pudo responder "No sabe/No contesta", siendo estas opciones consideradas como casos perdidos.

\subsection{ANÁLISIS ESTADÍSTICO}

Se realizó un análisis de Kolmogorov-Smirnov con el paquete estadístico SPSS 22.0 para conocer el comportamiento de los datos. Tras la confirmación del comportamiento normal de los mismos, se utilizó un modelo lineal general de medidas repetidas para analizar el efecto de la intervención en cada uno de los grupos (intervención y control) al inicio y al final del proceso de enseñanza-aprendizaje sobre la percepción de competencias de evaluación y la motivación del alumnado en las clases de EF. Las comparaciones por pares se realizaron utilizando el ajuste de Bonferroni y el tamaño del efecto se calculó con la eta parcial al cuadrado $\left(\eta_{\mathrm{p}}{ }^{2}\right)$. Valores de la $\eta_{\mathrm{p}}{ }^{2}$ mayores a 0,14 , entre $0,14-0,06$, entre 0,06 0,01 y menores a 0,01 informan de efectos grandes, moderados, pequeños y arbitrarios, respectivamente (Cohen, 1988).

Además, se utilizaron tablas de contingencia y el estadístico chi-cuadrado $\left(\chi^{2}\right)$ para analizar el grado de acuerdo del alumnado en el planteamiento docente de la asignatura según si pertenecían al grupo control o intervención $(p<0,05)$. En este caso, el tamaño del efecto se calculó con el coeficiente de contingencia de Pearson (CC).

\section{RESULTADOS}

El análisis comparativo mostró un efecto principal de la intervención en EFC en la motivación del alumnado $\left(\mathrm{F}_{(1,332)}=61,11 ; p<0,001 ; \eta_{\mathrm{p}}{ }^{2}=0,12\right)$, pero no en la percepción de competencias de evaluación en EF. Las comparaciones por pares se muestran en la Figura 1.

En cuanto a las cuestiones sobre el grado de acuerdo del alumnado en el planteamiento docente de la asignatura, cabe señalar que, en ambos grupos (control e intervención), la frecuencia de respuestas en las opciones no/nada o poco de acuerdo (valoradas entre 1 y 2 sobre 5) en todas las cuestiones fueron las menos elegidas de las cinco posibles respuestas, mostrándose así cierta tendencia hacia el grado de acuerdo del alumnado sobre el planteamiento propuesto. Además, los análisis comparativos antes de la intervención mostraron que únicamente en la cuestión sobre la negociación de la evaluación hubo 
diferencias en la frecuencia de respuesta entre los dos grupos, es decir, el grupo intervención mostró una menor proporción (24,5\% frente a un 75,5\% del grupo control) de alumnos y alumnas respondiendo algo de acuerdo (valorada con 3 sobre 5) y una mayor proporción (68\% frente a un $32 \%$ del grupo control) con muy de acuerdo (valorado con 5 sobre 5) que el grupo control $\left(\chi^{2}=30,33 ; p<0,001 ; C C=0,29\right)$. En el resto de cuestiones sobre la metodología, el sistema de evaluación para la adquisición de competencias, el grado de satisfacción y la utilidad de los contenidos propuestos, no se encontraron diferencias entre los grupos $(p>0,05)$. Respecto a los resultados después de la intervención, se halló que en la mayoría de respuestas sobre la negociación $\left(\chi^{2}=54,07 ; p<0,001 ; \mathrm{CC}=0,37\right)$, la metodología propuesta $\left(\chi^{2}=53,59 ; p<0,001 ; \mathrm{CC}=0,37\right)$, el sistema de evaluación $\left(\chi^{2}=\right.$ $44,91 ; p<0,001 ; \mathrm{CC}=0,34)$, el grado de satisfacción $\left(\chi^{2}=58,80 ; p<0,001 ; \mathrm{CC}=0,38\right) \mathrm{y}$ la utilidad de los contenidos propuestos de la asignatura $\left(\chi^{2}=43,84 ; p<0,001 ; \mathrm{CC}=0,34\right)$, el grupo intervención presentó una mayor proporción de respuestas bastante de acuerdo y muy de acuerdo (valoradas entre 4 y 5 sobre 5) que el grupo control (ver Tabla 1).

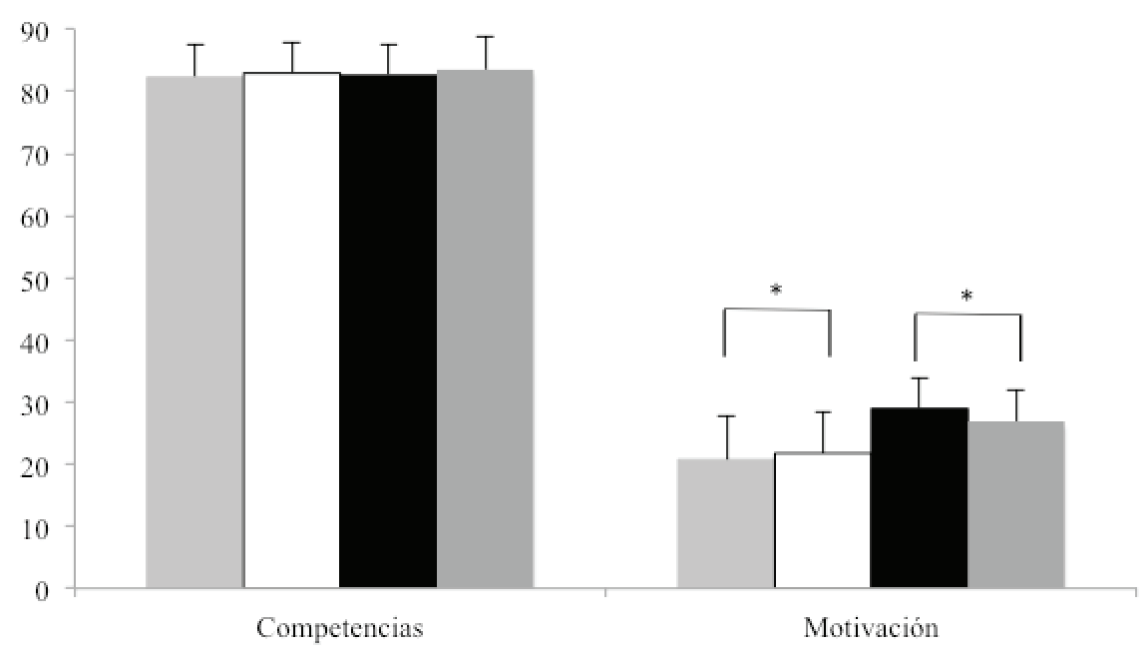

Figura 1. Estadísticos descriptivos y comparativos de la percepción de competencias en evaluación (rango 22 - 110) y motivación (rango 8 - 40) en EF antes y después de la intervención tanto para el grupo intervención como el control. Las barras gris claro y blanco se refieren al grupo intervención antes y después, respectivamente. Las barras negras y gris oscuro se refieren al grupo control antes y después, respectivamente. ${ }^{*}$ se refiere a diferencias significativas $(p<0,05)$. 
Estudios Pedagógicos XLIV, N 2: 205-221, 2018

EFECTO DE LA INTERVENCIÓN DOCENTE EN LA PERCEPCIÓN DE COMPETENCIA Y MOTIVACIÓN DE FUTUROS MAESTROS DE PRIMARIA EN EDUCACIÓN FÍSICA UTILIZANDO LA EVALUACIÓN FORMATIVA Y COMPARTIDA

Tabla 1. Frecuencia de respuestas de las y los participantes sobre el grado de acuerdo en el planteamiento docente de la asignatura después de la intervención

\begin{tabular}{|l|c|c|c|c|c|c|c|c|c|c|c|c|}
\hline & \multicolumn{2}{|c|}{ Ns/Nc } & \multicolumn{2}{c|}{ No/nada } & \multicolumn{2}{c|}{ Poco } & \multicolumn{2}{c|}{ Algo } & \multicolumn{2}{c|}{ Bastante } & \multicolumn{2}{c|}{ Mucho } \\
\hline & Interv & Control & Interv & Control & Interv & Control & Interv & Control & Interv & Control & Interv & Control \\
\hline Negociación & 4,5 & 8,7 & $9 *$ & 21,8 & $5,2^{*}$ & 13,1 & $9,7 *$ & 17,5 & 29,1 & 28,2 & $42,5^{*}$ & 10,7 \\
\hline Metodología & 0 & 0 & 0,7 & 0,5 & $0 *$ & 5,8 & $4,5^{*}$ & 27,7 & 56,0 & 52,4 & $38,8^{*}$ & 13,6 \\
\hline Evaluación & 1,5 & 0 & 0 & 0,5 & $2,2^{*}$ & 8,3 & $8,2^{*}$ & 33,5 & $60,4^{*}$ & 45,6 & $27,6^{*}$ & 12,1 \\
\hline Satisfacción & 0,7 & 0,5 & 0 & 1,9 & 3 & 6,3 & $6,0^{*}$ & 32,5 & 47 & 45,1 & $43,3 *$ & 13,6 \\
\hline Utilidad & 0 & 0 & 0 & 0,5 & 1,5 & 3,4 & $2,2 *$ & 19,9 & 47,0 & 55,8 & $49,3 *$ & 20,4 \\
\hline
\end{tabular}

Nota. Los valores se expresan en porcentaje de respuestas respecto al grupo al que pertenecen, intervención o control. Interv se refiere a intervención. Negociación se refiere a la negociación del sistema de evaluación. Metodología se refiere a la metodología docente. Evaluación se refiere a la adquisición de competencias en evaluación. Satisfacción se refiere al grado de satisfacción. Utilidad se refiere a la utilidad de los contenidos trabajados. * se refiere a diferencias significativas con el grupo control $(p<0,05)$.

\section{DISCUSIÓN Y CONCLUSIONES}

El objetivo del presente trabajo fue analizar el efecto que sobre el alumnado perteneciente al Grado en Maestro o Maestra de Educación Primaria tuvo una intervención docente basada en EFC. Grosso modo, podemos destacar que el alumnado que ha participado en la EFC ha mostrado un aumento en su motivación hacia los contenidos, en este caso de EF. Este hecho constituye uno de los fundamentos para que las y los defensores de una evaluación alternativa la reivindiquen con más ahínco, siendo numerosos los estudios en los que se arrojan datos en esta dirección (Atienza et al., 2016; Fraile \& Cornejo, 2012; Hortigüela, Pérez-Pueyo, \& Abella, 2015). Recientemente, Atienza et al. (2016) han coincidido en que el alumnado se muestra muy satisfecho con la participación en EFC, teniendo en cuenta todos los ítems, incluyendo la motivación. En esta dirección, Fraile y Cornejo (2012) obtuvieron datos similares, haciendo hincapié en la utilidad que este tipo de experiencias tiene para las y los futuros maestroas. Por su parte, Hortigüela PérezPueyo y Abella (2015) concluyen que la EFC obtiene mejores valoraciones por parte del alumnado en comparación con los sistemas tradicionales de evaluación, donde se destaca la implicación, los aprendizajes obtenidos o las responsabilidades asumidas.

Si entramos en detalle, vemos cómo el análisis comparativo también muestra que no hay un efecto de la intervención en la percepción de competencias docentes en evaluación. Ambos grupos, intervención y control, perciben que tienen un alto nivel de competencias en evaluación docente en EF (puntuando alrededor de 80 sobre 110). Estos datos contradicen a los mostrados por Hortigüela et al. (2016), quienes demostraron que la participación en EFC producía mayor percepción de adquisición de las competencias en comparación con aquellos y aquellas que habían seguido una evaluación tradicional. Nuestros resultados concuerdan con los de estudios que han puesto de manifiesto la dificultad de desarrollar un sistema de competencias en el aula, entre otras cosas, por las barreras diversas que encuentra 
el profesorado para ello (Zapatero-Ayuso et al., 2017). La falta de guías y directrices claras hace que la programación sea la tradicional, donde las competencias se suman, pero no son el centro de atención a la hora de programar (Figueras et al., 2016). Y es que, tomando las palabras de Blázquez (2010), evaluar por competencias no resulta fácil, pues hay que descender desde su formulación abstracta hasta las tareas de evaluación concretas; quizás, en forma de autocrítica, las tareas diseñadas durante la intervención no mantuvieron dicha formulación, aspecto que se debería haber considerado previamente.

Como consecuencia de lo anterior, no se ha conseguido uno de los objetivos deseados (i.e., mejorar la percepción de competencia en evaluación en EF del alumnado del grupo intervención). Como ya hemos detallado más arriba, parte del alumnado cursaba tercer curso, es decir, ya había escogido la mención de EF, mientras que otra parte de la muestra estaba todavía en segundo, aunque cursando una asignatura relacionada con esta área. En este sentido, para explicar la falta de una mejora percibida en las competencias docentes en evaluación en ambos grupos (intervención y control), siguiendo los comentarios de Hortigüela, Pérez-Pueyo y Fernández-Rio (2015), podríamos aludir al hecho de que el alumnado en cuestión, por su formación previa, ya dispone de una experiencia variada en metodologías y formas de evaluación impartidas en otras asignaturas. Este hecho explicaría la alta puntuación que marcan ambos grupos en el test inicial. Esto nos lleva a pensar que la participación en EFC y las metodologías activas propuestas no han tenido la profundidad necesaria como para provocar un impacto en el alumnado. A pesar de que se ha de tener en cuenta que cada experiencia y su contexto son peculiares, lo que puede afectar a los resultados, también hemos de indicar algunas posibles limitaciones de la intervención respecto a los contenidos valorados. En primer lugar, ponemos encima de la mesa el concepto de "alineación curricular" de Biggs (2008), con el objeto de iniciar el argumento por el cual, quizás en relación con la evaluación, no hemos planteado una metodología docente lo suficientemente activa, sobre todo teniendo en cuenta el punto de partida del alumnado participante. Cabe señalar que no se preguntó el nivel de conocimiento y experiencia previa en EFC del alumnado del grupo intervención, cuestión que podría afectar a su percepción con relación a la adquisición de competencias en evaluación debido a los matices que puedan diferenciar el presente planteamiento con lo anteriormente experimentado (e.g., debido a la naturaleza de los contenidos de EF frente a otras áreas). Además, poner el foco de atención únicamente en la evaluación para desarrollar una EFC puede hacernos prestar menos atención a otros elementos curriculares que también pueden influir en las competencias en evaluación, como la metodología (e.g., metodología cooperativa). Por ello, aunque el alumnado valore positivamente la participación en EFC, no ha percibido cómo la intervención podría mejorar sus competencias docentes en evaluación. Esto nos hace recordar el argumento de Lorente y Kirk (2012) en alusión a que el desarrollo de una evaluación alternativa no se circunscribe únicamente a la adopción de nuevas técnicas. La forma en que planteamos la evaluación debe ser coherente con el resto de elementos curriculares (Bretones, 2002). En esta dirección, por ejemplo, sirva el caso de Fraile y Cornejo (2012), quienes sumaron a la EFC métodos activos como el aprendizaje cooperativo o la resolución de problemas. La falta de alineación, por el contrario, puede hacer que el alumnado vea la EFC como un añadido al resto de elementos (Martos-García et al., 2017), obviando su importancia y sus beneficios. La combinación de propuestas o la hibridación de modelos pedagógicos parece dar buenos resultados educativos, por ejemplo, usando el aprendizaje cooperativo (Fernández-Rio \& Méndez-Giménez, 2016). Por ello, 
de acuerdo con los resultados de Fraile y Cornejo (2012), pensamos que combinarlo con la EFC podría favorecer la adquisición de competencias de los futuros egresados y egresadas.

Otra de las explicaciones que se pueden adoptar para explicar la razón por la cual el alumnado participante no ha mejorado sino mantenido una percepción similar en sus competencias docentes en evaluación, sería la que alude al principio de sostenibilidad. De acuerdo a López-Pastor (2009b), la EFC necesita ser viable, teniendo en cuenta las condiciones de trabajo, las circunstancias personales y otros factores. Así, estas iniciativas requieren de cierta atención por parte del profesorado, por ejemplo, evaluando continuamente las tareas que pide al alumnado para, de esta forma, proporcionar el feedback necesario para que se den procesos de evaluación formativa. Por ello, en grupos con 45 personas matriculadas es complicado hacer un seguimiento con las mínimas garantías, por lo que, a la hora de diseñar la EFC, hemos de atender a este hecho.

En lo que respecta a las puntuaciones al final de la intervención, se observa que hay algunas diferencias en ambos grupos. Sorprendentemente, el efecto de las propuestas docentes (intervención y control) es dispar, es decir, en el grupo de intervención en EFC la motivación del alumnado aumenta tras el periodo docente, lo que apoya lo sugerido por Moreno-Murcia et al. (2008, 2009) en tanto los participantes del grupo intervención entienden la mejora personal en tareas concretas, el esfuerzo y la perseverancia como un aspecto prioritario para la función docente, frente a la mera comparación con los demás en los procesos de evaluación en EF. Por el contrario, en el grupo control, la motivación por los contenidos de EF disminuye. Consecuentemente, parece ser que incluir la EFC en contenidos relacionados con la EF favorece la motivación de los futuros egresados, y ello puede ser visto como una forma de que, en su futuro profesional, si finalmente se ven en la situación de tener que impartir sesiones de EF, puedan recordar y tener presente experiencias positivas que favorezcan su orientación docente (López-Pastor \& SiciliaCamacho, 2017).

Tal y como hemos señalado en el apartado de procedimiento, al alumnado del grupo de intervención se le ofreció la posibilidad de negociar con el profesorado respectivo los porcentajes de calificación de cada ítem de evaluación. Este hecho va en la línea de hacer partícipes a las y los alumnos de cuantos más elementos mejor, permitiendo su participación en la evaluación y sin cerrarles la puerta cuando se trata de calificar, como sugiere Santos Guerra (1993). Sin embargo, los ítems de evaluación no estuvieron sujetos a la negociación, sino que estaban preestablecidos por el profesorado. Este hecho, que Biggs (2008) etiqueta como la forma más conservadora de EFC, sí ha arrojado resultados interesantes sobre el grado de acuerdo del alumnado en el planteamiento docente de la asignatura. Antes de la intervención (tras la explicación del planteamiento docente), el grupo intervención expresó con mayor frecuencia estar muy de acuerdo con la negociación de la evaluación. Tras la intervención, la diferencia en la frecuencia de respuesta se hizo más notable si cabe, encontrando una menor proporción de respuestas 'nada o poco de acuerdo' del grupo intervención frente al control y manteniendo las diferencias iniciales en 'muy de acuerdo', a favor del grupo intervención. Es decir, al finalizar la experiencia docente el alumnado del grupo de intervención sí es consciente del planteamiento experimentado (Tabla 1). De todas formas, para dar mayor calado a la experiencia vivenciada, parece pertinente en el futuro someter una mayor parte del sistema de evaluación a la negociación con el alumnado.

En relación con la metodología utilizada, pese a que antes del planteamiento docente no se encontraron diferencias en la proporción de respuestas entre ambos grupos, tras la 
experiencia docente el grupo intervención respondió con mayor frecuencia estar muy de acuerdo (valorando 5 sobre 5) en que el sistema de evaluación y la metodología utilizada (Tabla 1) les había ayudado a adquirir competencias profesionales. Por otro lado, el grupo control respondió con más frecuencia que les había ayudado poco o algo (valorando 2 o 3 sobre 5), frente al grupo intervención. Además, a diferencia que al principio, tras la intervención, el grado de satisfacción del grupo intervención fue con mayor frecuencia muy de acuerdo que el grupo control. En esta línea, el alumnado del grupo intervención también respondió con mayor frecuencia como 'muy de acuerdo' que la experiencia les había sido útil para el aprendizaje de contenidos de la asignatura. Aunque inicialmente estos resultados y los anteriores respecto a la no existencia de mejora en la percepción de competencias en evaluación (Figura 1) podrían parecer contradictorios, cabe destacar que los futuros egresados parecen considerar como apropiada la inclusión de EFC en su formación y ésta genera ciertos efectos en el grado de acuerdo del alumnado en el planteamiento docente de la asignatura.

Como se puede apreciar en el presente trabajo, únicamente se ha valorado la percepción del alumnado en las competencias, la motivación y la impresión del proceso de enseñanzaaprendizaje. Las escalas utilizadas para el presente estudio han mostrado una alta consistencia interna en las respuestas de los participantes. Sin embargo, aunque la escala de motivación ha sido validada en el ámbito de la EF y sus creadores sugieren su aplicación en el profesorado del área, hasta la fecha, su utilización se ha centrado principalmente en alumnado de ESO. También cabe señalar que aunque la escala de competencias docentes en evaluación fue revisada por expertos en la materia, no ha seguido un proceso de revisión de expertos ciegos sin vinculación en el presente estudio. Así pues, futuros estudios deberían afrontar el análisis de la validación de las escalas utilizadas para garantizar su utilización apropiada en el presente contexto universitario.

Además, no se ha tenido en cuenta el éxito en la superación de las asignaturas impartidas. Pese a que es característica común en la titulación de Maestro o Maestra en Educación Primaria que el porcentaje de éxito sea elevado, sería necesario que futuros estudios analizaran la relación entre la percepción del alumnado y el éxito académico durante intervenciones docentes utilizando EFC. De igual manera, parece pertinente, aunque las percepciones del alumnado sean tremendamente importantes, que dichos datos se crucen con las percepciones del profesorado. Este tipo de estudios permite contrastar ambas visiones para, de esta forma, establecer similitudes y diferencias en la interpretación de los procesos vividos y extraer conclusiones de ello (Brew et al., 2009).

A modo de conclusión, a la vista de los resultados, se observa que una intervención docente centrada no solamente en una metodología activa y participativa, sino también en la inclusión de la EFC, favorece que el alumnado del Grado de Maestro o Maestra en Educación Primaria mejore su motivación hacia los contenidos de la asignatura de EF, estableciéndose una relación causal. Por el contrario, cuando no se incluye la EFC, la motivación disminuye. En ambos casos, exista o no EFC, se confirma una similar percepción de competencias en evaluación antes y después del planteamiento docente.

Desde un punto de vista aplicado, se sugiere la inclusión de la EFC en los planteamientos docentes, ya que en el alumnado se da un mayor nivel de acuerdo con fórmulas características de la EFC como, por ejemplo, la negociación de la evaluación, la metodología seguida, la adquisición de competencias, el grado de satisfacción y la utilidad de los contenidos de la asignatura de EF. 
Estudios Pedagógicos XLIV, N $^{\circ}$ 2: 205-221, 2018

EFECTO DE LA INTERVENCIÓN DOCENTE EN LA PERCEPCIÓN DE COMPETENCIA Y MOTIVACIÓN DE FUTUROS

MAESTROS DE PRIMARIA EN EDUCACIÓN FÍSICA UTILIZANDO LA EVALUACIÓN FORMATIVA Y COMPARTIDA

\section{REFERENCIAS BIBLIOGRÁFICAS}

Atienza, R., Valencia-Peris, A., Martos-García, D., López-Pastor, V.M., \& Devís-Devís, J. (2016). La percepción del alumnado universitario de educación física sobre la evaluación: Ventajas, dificultades y satisfacción. Movimento, 22(4), 1033-1048.

Berk, R.A. (2005). Survey of 12 Strategies to Measure Teaching Effectiveness. International Journal of Teaching and Learning in Higher Education, 17(1), 45-62.

Biggs, J. (2008). Calidad del aprendizaje universitario (3a ed.). Madrid: Narcea.

Blázquez, D. (2010). La evaluación de las competencias en educación física. En D. Blázquez y E. Sebastiani (Eds.), Enseñar por competencias en Educación Física (pp. 163-190). Barcelona: Inde.

Boud, D., \& Falchikov, N. (2007). Rethinking assessment in higher education. Learning for the long term. Londres: Routledge.

Bretones, A. (2002). La participación del alumnado en la evaluación de sus aprendizajes. Kikiriki, 65, 6-15.

Brew, C., Riley, P., \& Walta, C. (2009). Education students and their teachers: comparing views on participative assessment practices. Assessment \& Evaluation in Higher Education, 34(6), 641657. doi:10.1080/02602930802468567

Brockbank, A., \& McGill, I. (2002). Aprendizaje reflexivo en la educación superior. Madrid: Morata.

Brown, S., \& Glasner, A. (2003). Evaluar en la Universidad: problemas y nuevos enfoques. Madrid: Narcea.

Carless, D., Joughin, G., \& Mark, M. M. C. (2006). Learning-oriented assessment: principles and practice. Assessment \& Evaluation in Higher Education, 31, 395-398. doi:10.1080/02602930600679043

Carson, R. L., \& Chase, M. A. (2009). An examination of physical education teacher motivation from a self-determination theoretical framework. Physical Education and Sport Pedagogy, 14(4), 335-353. doi:10.1080/17408980802301866

Chen, W. (2005). Examination of curricula, teaching practices, and assessment through national standards.PhysicalEducationandSportPedagogy, 10,159-180.doi:10.1080/17408980500105056

Cohen, J. (1988). Statistical power analysis for the behavioural sciences (2a ed.). Hillsdale, NJ: Lawrence Erlbaum.

Dekker-Groen, A., Van der Schaaf, M., \& Stokking, K. (2013). A Teacher Competence Development Programme for Supporting Students' Reflection Skills. Teachers and Teaching: Theory and Practice, 19(2), 150-171.

Diario Oficial de la Unión Europea. (20 de diciembre de 2006). Recomendación del Parlamento Europeo y del Consejo de 18 de diciembre de 2006 sobre las competencias clave para el aprendizaje permanente, 394/10-394/18. Recuperado de http://eur-lex.europa.eu/legal-content/ ES/TXT/PDF/?uri=CELEX: 32006H0962\&from $=\mathrm{EN}$

Dochy, F., Segers, M., \& Dierick, S. (2002). Nuevas vías de aprendizaje y enseñanza y sus consecuencias: una era de evaluación. Revista de Docencia Universitaria, 2(2), 13-30.

Fernández-Rio, J., \& Méndez-Giménez, A. (2016). El Aprendizaje Cooperativo: Modelo Pedagógico para Educación Física. Retos, 29, 201-206

Figueras, S., Capllonch, M., Blázquez, D., \& Monzonís, N. (2016). Competencias básicas y educación física: estudios e investigaciones. Apuntes. Educación Física y Deportes, 123, 34-43.

Fraile, A., \& Cornejo, P. (2012). La evaluación formativa en la enseñanza universitaria: una experiencia de innovación educativa con estudiantes de Educación Física. Revista de evaluación educativa, 1(2). Recuperado de http://revalue.mx/revista/index.php/revalue/issue/current

Hamodí, C. (2016). Terminología básica sobre evaluación del aprendizaje. En C. Hamodí (Coord.), Formar mediante la evaluación en la universidad. Propuestas prácticas útiles para docentes (pp. 23-32). Valladolid: Ediciones de la Universidad de Valladolid. 
Hortigüela, D., Abella, V., \& Pérez-Pueyo, A. (2015). ¿Se Han Implantado las Competencias Básicas en los Centros Educativos? Un Estudio Mixto sobre su Programación como Herramienta de Aprendizaje. Revista Iberoamericana de Evaluación Educativa, 8(1), 177-192.

Hortigüela, D., Pérez-Pueyo, A., \& Abella, V. (2015). ¿De qué manera se implica el alumnado en el aprendizaje? Análisis de su percepción en procesos de evaluación formativa. Revista de Investigación en Educación, 13(1), 88-104.

Hortigüela, D., Pérez-Pueyo, A., \& Fernández-Rio, J. (2015). Influencia de las experiencias vivenciadas por el alumnado en el desempeño de futuras competencias docentes. Contextos Educativos, 19, 25-41.

Hortigüela, D., Pérez-Pueyo, A., \& López-Pastor, V. M. (2015). Implicación y regulación del trabajo del alumnado en los sistemas de evaluación formativa en educación superior. Relieve: Revista Electrónica de Investigación y Evaluación Educativa, 21(1), 1-5. doi:10.7203/relieve.21.1.5171

Hortigüela, D., Abella, V., Delgado, V., \& Ausín, V. (2016). Influencia del sistema de evaluación empleado en la percepción del alumno sobre su aprendizaje y las competencias docentes. Infancia, Educación y Aprendizaje, 2(1), 20-42.

Ley Orgánica 2/2006, de 3 de mayo, de Educación. Boletín Oficial del Estado de 4 de mayo de 2006.

Ley Orgánica 8/2013, de 9 de diciembre, para la mejora de la calidad educativa. Boletín Oficial del Estado de 10 de Diciembre de 2013.

López Pastor, V. M. (2008). Desarrollando sistemas de evaluación formativa y compartida en la docencia universitaria. Análisis de resultados de su puesta en práctica en la formación inicial del profesorado. European Journal of Teacher Education, 31(3), 293-311. doi:10.1080/02619760802208452

López-Pastor, V. M. (2009a). Fundamentación teórica y revisión del estado de la cuestión. En V. M. López-Pastor (Coord.), Evaluación Formativa y Compartida en Educación Superior. Propuestas, técnicas, instrumentos y experiencias (pp. 45-64). Madrid: Narcea.

López-Pastor, V. M. (2009b). Una propuesta genérica de evaluación formativa y compartida para la docencia universitaria. En V. M. López-Pastor (Coord.), Evaluación Formativa y Compartida en Educación Superior. Propuestas, técnicas, instrumentos y experiencias (pp. 93-103). Madrid: Narcea.

López-Pástor, V. M. (2011). El papel de la evaluación por competencias: aportaciones de la red de evaluación formativa y compartida en docencia universitaria. Revista de Docencia Universitaria, 9, 159-173. doi:10.4995/redu.2011.6185

López-Pastor, V. M., Castejón, J., Sicilia-Camacho, A., Navarro-Adelantado, V., \& Webb. G. (2011). The process of creating a cross-university network for formative and shared assessment in higher education in Spain and its potential applications. Innovations in Education and Teaching International, 48(1), 79-90. doi:10.1080/14703297.2010.543768

López-Pastor, V. M., Kirk, D., Lorente-Catalán, E., MacPhail, A., \& Macdonald, D. (2013a). Alternative assessment in physical education: a review of international literature. Sport, Education and Society, 18(1), 57-76. doi:10.1080/13573322.2012.713860

López-Pastor, V. M., Pintor, P., Muros, B., \& Webb, G. (2013b). Formative assess-ment strategies and their effect on student performance and on student and tu-tor workload: the results of research projects undertaken in preparation for greater convergence of universities in Spain within the European Higher Edu-cation Area (EHEA). Journal of Further and Higher Education, 37(2), 163-180. doi:10.1080/0309877X.2011.644780

López-Pastor, V.M., \& Sicilia-Camacho, A. (2017). Formative and shared assessment in higher education. Lessons learned and challenges for the future, Assessment \& Evaluation in Higher Education, 42(1), 77-97. doi:10.1080/02602938.2015.1083535

López-Pastor, V. M., Monjas, R., Gómez, J., López-Pastor, E. M., Martín, J. F., González, J., . . . Marugán, L. (2006). La evaluación en educación física. Revisión de modelos tradicionales y planteamiento de una alternativa. La evaluación formativa y compartida. Retos. Nuevas 
Estudios Pedagógicos XLIV, N $^{\circ}$ 2: 205-221, 2018

EFECTO DE LA INTERVENCIÓN DOCENTE EN LA PERCEPCIÓN DE COMPETENCIA Y MOTIVACIÓN DE FUTUROS MAESTROS DE PRIMARIA EN EDUCACIÓN FÍSICA UTILIZANDO LA EVALUACIÓN FORMATIVA Y COMPARTIDA

Tendencias en Educación Física, Deporte y Recreación, 10, 31-41.

Lorente, E., \& Kirk, D. (2012). Alternative democratic assessment in PETE: an action-research study exploring risks, challenges and solutions. Sport, Education and Society, 1(20), 77-96. doi:10.10 80/13573322.2012.713859

Martos-García, D., Usabiaga, O., \& Valencia-Peris, A. (2017). Students' Perception on Formative and Shared Assessment: Connecting two Universities through the Blogosphere. Journal of New Approaches in Educational Research, 6(1), 64-70. doi:10.7821/naer.2017.1.194

Méndez, A., López-Téllez, G., \& Sierra, B. (2009). Competencias Básicas: sobre la exclusión de la competencia motriz y las aportaciones desde la Educación Física. Retos. Nuevas tendencias en Educación Física, Deporte y Recreación, 16, 51-57.

Mintah, J. K. (2003). Authentic assessment in physical education: prevalence of use and perceived impact on students' self-concept, motivation, and skill achievement, Measurement in Physical Education and Exercise Science, 7(3), 161-174. doi:10.1207/S15327841MPEE070303

Monarca, H., \& Rappoport, S. (2013). Investigación sobre los procesos de cambio educativo: El caso de las competencias básicas en España. Revista de Educación, Extraordinario, 54-78. doi:10.4438/1988-592X-RE-2013- EXT-256

Moreno-Murcia, J. A., González-Cutre, D., \& Chillón, M. (2009). Preliminary Validation in Spanish of a Scale Designed to Measure Motivation in Physical Education Classes: The Perceived Locus of Causality (PLOC) Scale. The Spanish Journal of Psychology, 12, 327-337.

Moreno, J. A., González, D., Chillón, M., \& Parra, N. (2008). Adaptación a la educación física de la escala de las necesidades psicológicas básicas en el ejercicio. Revista Mexicana de Psicología, 25(2), 295-303.

Muros, B. (2013). Autoevaluación: bases epistemológicas y autoconocimiento. Una experiencia en el aula universitaria. Revista de evaluación educativa, 2(1). Recuperado de http://revalue.mx/ revista/index.php/revalue/issue/current

Palacios-Picos, A., \& López-Pastor, V. M. (2013). Haz lo que yo digo pero no lo que yo hago: sistemas de evaluación del alumnado en la formación inicial del profesorado. Revista de Educación, 361, 279-305. doi:10.4438/1988-592X-RE-2011-361-143

Pascual, C. (1996). La evaluación de la Educación Física, esa desconocida. Revista de Educación Física: Renovar la Teoría y Práctica, 63, 5-8.

Pérez-Pueyo, A., Julián, J.A., \& López-Pastor, V.M. (2009). Evaluación formativa y compartida en el espacio europeo de educación superior (EEES). En V. M. López-Pastor (Coord.), Evaluación Formativa y Compartida en Educación Superior. Propuestas, técnicas, instrumentos y experiencias (pp. 19-44). Madrid: Narcea.

Santos-Guerra, M.A. (1993). La evaluación: un proceso de diálogo, comprensión y mejora. Archidona: Ediciones Aljibe.

Sebastiani, E., Blázquez, D., \& Barrachina, J. (2010). Concepto y naturaleza de las competencias. En D. Blázquez y E. Sebastiani (Eds.), Enseñar por competencias en Educación Física (pp. 39-62). Barcelona: Inde.

Siedentop, D., \& Tannehill, D. (2000). Developing teaching skills in physical education (4th ed.). Mountain View: Mayfield.

Sparkes, A. (1992). Breve introducción a los paradigmas de investigación alternativos en educación física. Perspectivas, 11, 29-33.

Tan, K. H. K. (2008). Qualitatively different ways of experiencing student self-assessment. Higher Education Research \& Development, 27(1), 15-29. doi:10.1080/07294360701658708

Trevitt, C., Brenan, E., \& Stocks, C. (2012). Evaluación y aprendizaje: ¿es ya momento de replantearse las actividades del alumnado y los roles académicos? Revista de Investigación Educativa, 30, 253-269. doi:10.6018/rie.30.2.153441

Vallerand, R. J., \& Rousseau, F. L. (2001). Intrinsic and extrinsic motivation in sport and exercise: A review using the hierarchical model of intrinsic and extrinsic motivation. In R. N. Singer, H. A. 
Hausenblas \& C. M. Janelle (Eds.), Handbook of Sport Psychology (2a ed., pp. 389-416). Nueva York: John Wiley \& Sons.

Van der Berg, I., Admiraal, W., \& Pilot, A. (2006). Peer assessment in university teaching: Evaluating seven course designs. Assessment \& Evaluation in Higher Education, 31(1), 19-36. doi:10.1080/02602930500262346

Varsavsky, C., Matthews, K., \& Hodgson, Y. (2014). Perceptions of Science Graduating Students on Their Learning Gains. International Journal of Science Education, 36(6), 929-951. doi:10.108 0/09500693.2013.830795

Vicente, M. (2011). Escuela y educación física en el contexto de la enseñanza por competencias. Reflexiones genealógicas desde la pedagogía crítica. Cultura, Ciencia y Deporte, 18(6), 161170.

Zapatero-Ayuso, J., González-Rivera, M.D., \& Campos-Izquierdo, A. (2017). Dificultades y apoyos para enseñar por competencias en Educación Física en secundaria: un estudio cualitativo. Revista Internacional de Ciencias del Deporte, 13(47), 5-25. doi:10.5232/ricyde2017.04701 
